\title{
Design and Testing of a Thermoelectrically- Cooled Portable Vaccine Cooler
}

\author{
Elliot Reid ${ }^{*}$, Jared Barkes ${ }^{1}$, Cameron Morrison ${ }^{1}$, Andy Ung ${ }^{1}$, Roshni Patel ${ }^{1}$, Chase Rebarker ${ }^{1}$, Parth \\ Panchal $^{1}$, Sahil Vasa ${ }^{1}$
}

Improper handling and the failure to maintain vaccines in a safe temperature range results in significant losses of vaccines in the rural developing world. This issue has reduced the success of immunization programs in the developing world, and consequently confidence in vaccinations. For vaccine refrigeration and delivery at the end stage of the cold chain, active thermoelectric cooling is a potentially viable alternative because the coolers are small and require few or no moving parts. A vaccine cooling system was constructed employing a Peltier-based thermoelectric chip (TEC) affixed to an aluminum block that holds the vaccine vials; a movable copper plate was interposed between the block and the TEC, acting as a thermal diode to prevent backflow of heat from the TEC into the vaccine holder. The heat was transported away from the hot side of the TEC to a remote heat exchanger. The aluminum block was contained in a heavily insulated plexiglass box equipped with a port on the top for transient removal of vaccine vials. With this cooler, vaccines can be maintained in the required temperature ranges of $4-8^{\circ} \mathrm{C}$ for a conservatively estimated 10 hours at $37^{\circ} \mathrm{C}$ on less than one full battery charge, while requiring less space than a common beach cooler. Since many parts of the rural developing world have sunny climates, the potential of the cooler's power requirement to be partially supplied via solar panels was also evaluated.

\section{INTRODUCTION}

Vaccines are a major economic cost and component of the worldwide battle against infectious disease. In 2011, UNICEF bought 2.5 billion doses of vaccines, and spent more than one billion dollars on vaccines (UNICEF, 2011). Within the category of develop-ment assistance for maternal and child health, donors spent $\$ 3.2$ billion on child vaccines in 2014 (Dieleman, Murray, \& Haaken-stad, 2015). Still, for children in developing countries, health care inequities are prominent, and access to preventative therapies and drugs is limited (Gates, 2012). One reason these countries lack enough vaccination coverage is due to insufficient cold storage (without freezing) in the vaccine supply chain (Humphreys, 2011). Breakdown of the vaccine cold chain is believed to be a major contributor to late 20th-century polio outbreaks in southern Africa (Schoub, \& Cameron, 1996). Many vaccines must be maintained in a temperature range of $2-8^{\circ} \mathrm{C}$ to remain potent. Indeed, previous studies have shown that cold chain wastage--due to the failure to maintain vaccines in a safe temperature range, the need to discard unused portions of opened vaccine vials, and improper handling

${ }^{1}$ Department of Cell Biology and Physiology, University of North Carolina at Chapel Hill, 103 S. Bldng Cb 9100, Chapel Hill, NC 27599

*To whom correspondence should be addressed: emsr2@yahoo.com

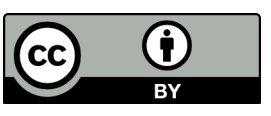

Except where otherwise noted, this work is licensed under https://creativecommons.org/licenses/by/4.0

doi:10.22186/jyi.35.2.50-55 and expiration --can be as high as $60-70 \%$ (Wallace, Willis, Nwaze, \& Dieng, 2017; Zaffran et al., 2013).

Although vaccine coolers are designed to keep vaccines cold, a poorly designed apparatus can result in accidental freezing of vaccines so that sub-potent vaccines can sometimes be administered (Chen, \& Kristensen, 2009). A 2007 study found that in vaccine reports tracked longitudinally, $75-100 \%$ of vaccines were exposed to freezing temperatures; the authors recommend im-proved cold-chain transport equipment as a solution (Matthias, Robertson, Garrison, Newland, \& Nelson, 2007). Vaccine freezing or overheating issues are not relegated solely to older studies or developing nations. The 2013-2014 H1N1pdm09 virus outbreak in the United States can likely be attributed to vaccine shipments being exposed to high temperatures (Caspard, Coelingh, Mallory, \& Ambrose, 2016).

The cost of most vaccines today ranges from $\$ 3.50-\$ 7.50$ per administration (Gates, 2012), so wastage results in a considerable economic loss. Importantly, when vaccines lose potency, there is a loss of confidence in vaccine therapy (Larson, Cooper, Eskola, Katz, \& Ratzan, 2011). Thus, reducing vaccine wastage while in-creasing potency will provide more effective immunization in the rural, developing world at a reduced cost per dose.

One way to address aspects of the wastage issue is the development of small coolers capable of transporting vaccines, maintained in the proper temperature range, from the regional health center to the distant client; this trip is termed the end stage of the cold chain. Coolers employing phase change materials including ice are capable of maintaining the desired temperature range for a period, but vaccines in such coolers are sometimes subject to overheating or freezing because of the lack of temperature regulation. 
A

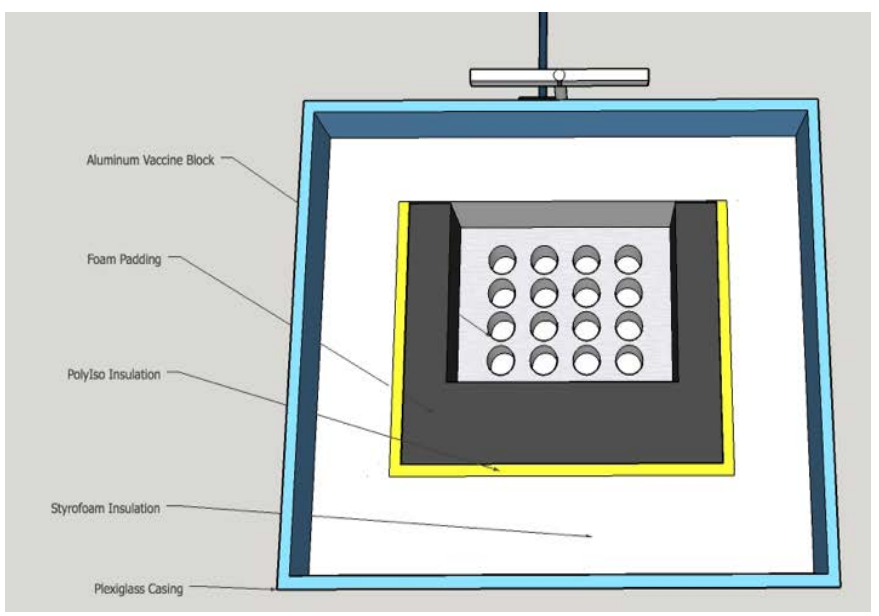

$\mathrm{C}$

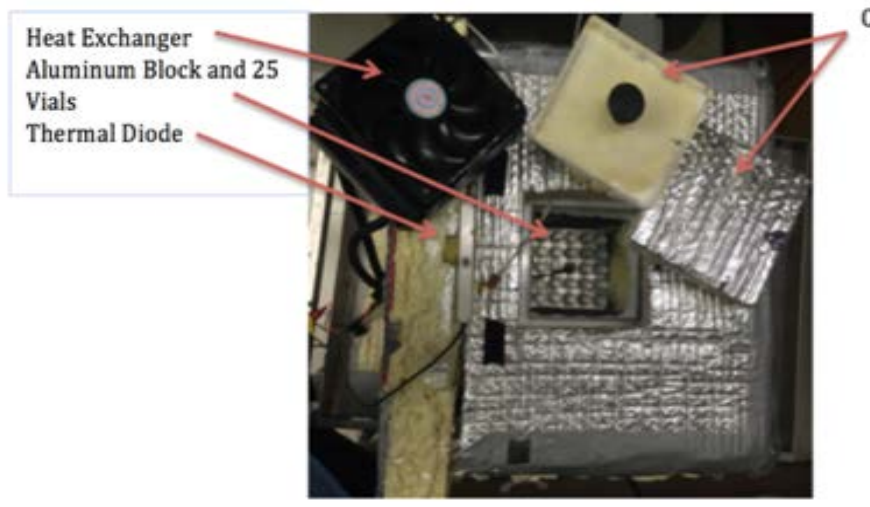

B

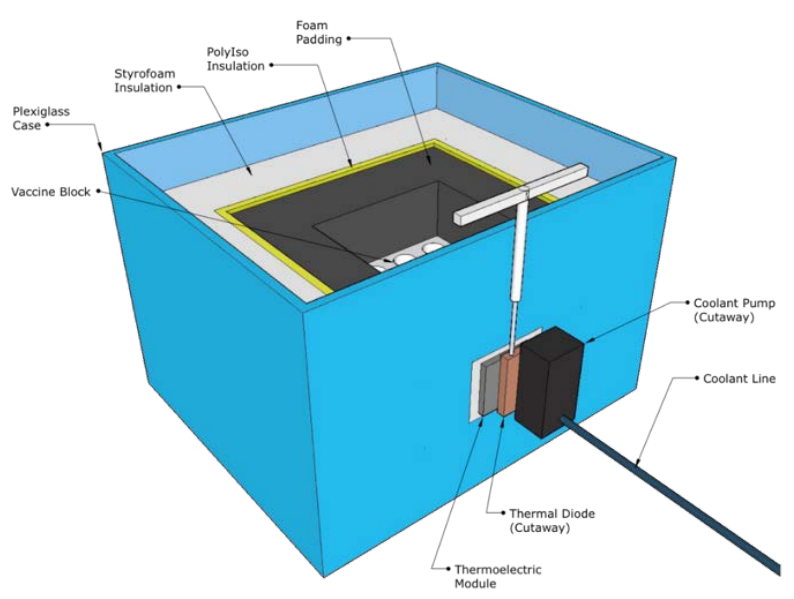

Cooler Top

D

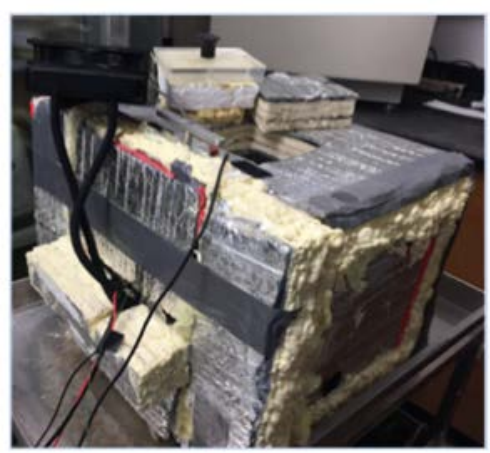

Figure 1. The Thermoelectric Vaccine Cooler. A) Schematic top view. B) Schematic oblique side view. C) Top view. D) Oblique side view image.

Solid-state thermoelectric cooling (Bell, 2008), employing the Peltier effect, offers an important alternate solution to temperatureregulated vaccine refrigeration (Ghosal, \& Guha, 2009). For small loads, thermoelectric coolers offer significant advantages compared to the more conventional vapor-compression refrigeration: there are far fewer moving parts that may require maintenance, no risks of refrigerant leakage, and a lighter, more compact size.

This paper provides a proof of concept for a small batterypowered, thermoelectrically cooled vaccine cooler to deliver safe vaccines. Several similar designs have been proposed previously (Chatterjee, \& Pandy, 2003; Ohara et al., 2014; Putra, 2009). By contrast to many previous studies that were conducted at room temperature, this cooler was tested with the express purpose of preserving vaccines at a high ambient temperature $\left(37^{\circ} \mathrm{C}\right)$, typical of temperatures that would be encountered in the tropical, rural developing world. Our results suggest that vaccines can be maintained in a safe temperature range for $\sim 10$ hours at an ambient temperature of $37^{\circ} \mathrm{C}$ with one battery charge; this is enough time in a working day to deliver vaccines from a regional distribution center to clients for administration and for the return trip to the center. Since many parts of the rural developing world have sunny climates, the potential for solar panels affixed to the sides of the cooler to recharge the on-board battery was explored.

\section{MATERIALS AND METHODS}

\section{System Design and Fabrication}

The design goal for the TE cooler was to maintain $125 \mathrm{~mL}$ of mock vaccine in twenty-five, $5 \mathrm{~mL}$ vials within a safe temperature range of $4-8^{\circ} \mathrm{C}$ for over 8 hours. The vaccines were held in an aluminum block with holes drilled to accommodate the vials (Figure 1A). The Al block is in direct contact with a TE Technology HP-1271.4-2.5-72 semiconductor thermoelectric chip (TEC, TE Technology Inc., Traverse City, MI) powered by a rechargeable RAVpower 23000mAh lithium-polymer battery (SunvalleyTek, Santa Clara, CA) wired directly to the leads on the TEC. By maintaining the cold side of the TEC at a temperature lower than the vaccines, heat flows from the vaccines to the TEC. The hot side of the TEC is in contact with a heat sink coupled to a Cooler Master Seidon 120V 


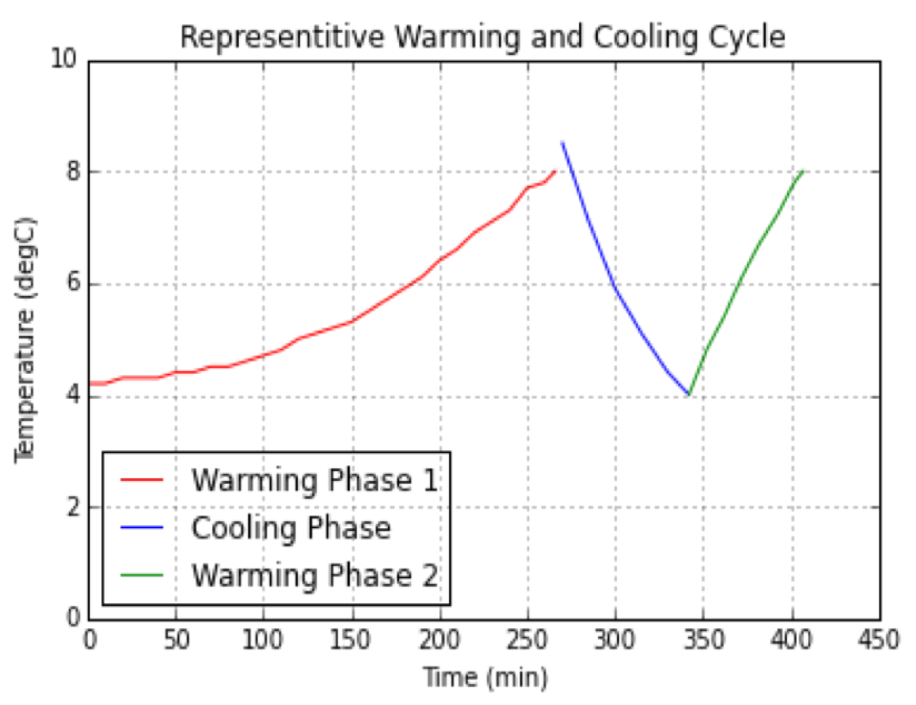

Figure 2. Vaccine temperature-time plot for a typical experiment.

remote heat exchanger (CoolerMaster Inc., Taipei). Between the Al block and cold side of the TEC is a manually removable copper slab that acts as a thermal diode (Figure 1B). The heat exchanger is powered by two smaller 2000mAh Lithium-Ion batteries (AllBattery.com, Fremont, California). Thermal grease, Thermaltake TG-7 (BestBuy, Richfield, MN), is used to ensure proper thermal contact between the interfaces of the hot and cold components.

The Al block is centered inside an 18"x18"x18" container having a plexiglass outer wall (thickness $1 / 2$ ") and insulated by layers of Styrofoam, polyisocyanurate panels and foam rubber placed between the plexiglass container and the Al block (Figure 1A, B). The outside of the box was covered with an additional 1.5" layer of Styrofoam. Reflectix foil (The Home Depot, Atlanta, GA) was attached to the outside of the box (Figure 1C, D). The top of the cooler box was fitted with a port with an insulated cover for transient vaccine removal; the side of the cooler has an exit for the fluid-containing tubes that transport heat from the heat sink affixed to the hot side of the TEM to an external heat exchanger. This exit port is filled with fiberglass wool insulation. The vaccine cooler weighs approximately $12 \mathrm{lbs}$. Polyurethane spray foam (The Home Depot, Atlanta, GA) was used to seal joints between the insulation components. Cool-packs (savENRG, Asheville, NC) were inserted between the insulation panels and the aluminum block to increase the thermal inertia of the system. A vaccine vial temperature monitor (Materro LLC, Potomac, MD) was inserted into the center vial hole in the $\mathrm{Al}$ holder and temperature reported with a digital readout. Schematic drawings and photographs of the system are shown in Figures 1A-D.

\section{System Testing}

Vaccine Cooler

An experiment consisted of a first warming phase, a cooling phase, and a second warming phase. Prior to the beginning of each experiment, the cooler was placed inside a refrigerator in order to bring the entire system down to an initial temperature of $4^{\circ} \mathrm{C}$. The cooler was then taken out of the refrigerator and placed inside of a warm room set at $37^{\circ} \mathrm{C}$ to simulate temperatures that may occur in the rural, developing world. The RAVpower 23000mAh lithiumpolymer battery was fully charged before beginning the experiment. The first warming time was defined as the time taken for the vaccines to warm from $4^{\circ} \mathrm{C}$ to $8^{\circ} \mathrm{C}$; the cooling time was defined as the time taken for the vaccines to cool from $8^{\circ} \mathrm{C}$ down to $4^{\circ} \mathrm{C}$ when the TEC and heat exchanger were turned on and the thermal diode (copper slab) was in place. The second warming time was initiated by removing the thermal diode and turning the TEC and heat exchanger off. In order to simulate field vaccine removal during the first warming phase, every 30 minutes the top port cover of the cooler was opened. Three vials of mock vaccine were removed and the top port cover was then replaced. These extractions were timed to take 15-30 seconds.

\section{Solar panel battery charging}

The potential for solar panels as a means to partially charge the onboard batteries was also investigated. Two EcoDirect 10-Watt Solarland Slp 010-124 solar panels (Ecodirect, Vista, CA), approximately the size of each side of the external cooler box, were placed in direct sunlight and attached to one of the two smaller $2000 \mathrm{mAh}$ Lithium-ion batteries (batteryspace.com, Richmond, CA) used to power the fan and pump of the heat exchanger subsystem. A solar charge controller (batteryspace.com, Richmond, CA) and a digital volt and current meter were placed in the charging circuit and the current and voltage produced by the panels was measured. Approximately every 10 minutes, readings for the voltage and current were recorded and the battery capacity was checked. The charging cable has two LED lights that indicate in binary fashion when the battery is fully charged: when the red LED light is lit the battery is still being charged and when the green LED is lit the battery is fully charged.

\section{RESULTS}

\section{Overall performance}

The result of a typical experiment is given in terms of temperature versus time plots shown in Figure 2 performed as described in the System Testing section. Typically, during each cooling phase, the TEC was operated at $3 \mathrm{~A}$ and $9 \mathrm{~V}$. The thermoelectric cooler was able to maintain vaccines in the target $4-8^{\circ} \mathrm{C}$ temperature range during a first and second warming phase for an average of 249 minutes and 65 minutes, respectively (Table 1 ). If the thermal diode is left in place during the first warming phase, additional heat is conducted in from the environment with the result that the first warming phase time is reduced to 135 minutes. Experimental variation in the first and second warming phases is given Figure $3 \mathrm{~A}$ and $3 \mathrm{C}$, respectively, while experimental variation in the first cooling phase is shown in Figure 3B. The similarity of slopes of the warming and cooling temperature-time data in Figure 3 indicates warming and cooling rates were similar in the different experiments. The lithium-ion battery provides the current when the TEC is on and cooling the vaccines. For each cooling phase, $\sim 400 \mathrm{mAh}$ of charge is drained from the battery or $\sim 20.4 \%$ of total battery 
A)

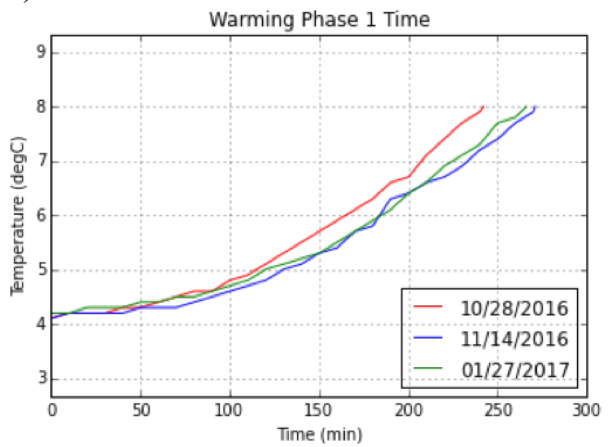

B)

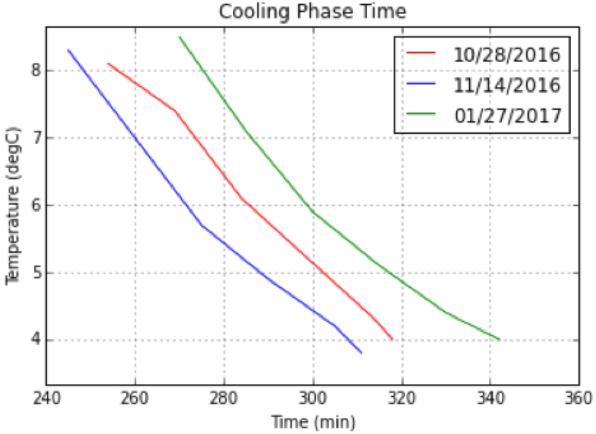

C)

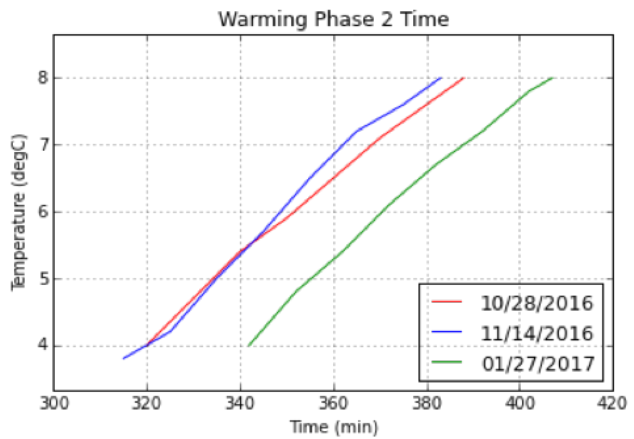

Figure 3. Representative vaccine temperature-time curves for individual warming and cooling curves. A) First warming phase. B) First cooling phase. C) Second warming phase. Three extractions were made in the warming phases. The temperature for the experiment was maintained at $37^{\circ} \mathrm{C}$ in a warm room.

capacity (Table 1$)$.

\section{Battery charging by solar panels}

The ability of the solar panels to charge the two smaller lithiumion batteries used to power the pump and fan of the heat exchanger subsystem was evaluated. The average charge supplied to the drained batteries from each solar panel was $332 \mathrm{mAh}$. When multiplied by the time of an average second warming phase, 1.08 hours, one battery was recharged $358.7 \mathrm{mAh}$. For one hour of operation, the pump and fan combined consume approximately 696mAh. When multiplied by the duration of an average cooling phase, 1.12 hours, one battery discharges $781 \mathrm{mAh}$. By extrapolation, two solar panels can recharge $717 \mathrm{mAh}$ during the second warming phase, greatly offsetting the battery consumption.

\section{DISCUSSION}

\section{Cooler Performance}

Generally, vaccines must be maintained in a narrow temperature range to avoid loss of potency. The range of $4-8^{\circ} \mathrm{C}$ was selected for compatibility with a broad spectrum of vaccines. In this range, the thermoelectric vaccine cooler described provides a viable solution to vaccine wastage due to lack of temperature control. Prior to a workday in the field, the battery powering the cooler would be charged overnight in the regional health center, and the TE cooler would be brought to $4^{\circ} \mathrm{C}$ before loading the vaccines. When the mean first and second warming phases (249 minutes and 65 minutes, respectively) are added to an average first cooling phase time of 67 minutes, the cooler could maintain vaccines in the target temperature range for just over 6 hours after one warming/cooling/warming cycle. The first warming phase from $4^{\circ} \mathrm{C}$ to $8^{\circ} \mathrm{C}$ can last over 4 hours because the system has maximum thermal inertia starting from a cold state. The second warming phase is much shorter than the first, presumably because much of the thermal inertia acquired when the entire cooler is cooled down to about $4^{\circ} \mathrm{C}$ prior to starting the experiment is lost when the cooler is maintained at $37^{\circ} \mathrm{C}$ for nearly 6 hours. Cooling below $4^{\circ} \mathrm{C}$ proved difficult with the current TEC when ambient temperatures were high (e.g. $37^{\circ} \mathrm{C}$, data not shown). Assuming subsequent cooling and warming phases each last roughly 60 minutes and a constant battery discharge rate, the cooler could preserve vaccines viable for well over 10 hours on a single battery charge. While working times could be reduced somewhat owing to the adverse effects of temperature and battery age on battery performance (Leng, Petch, $\&$ Tan, 2015), the cooler could be taken to the vaccine delivery site(s), clients served, and then brought back empty in an eighthour workday.

The cooling system is vulnerable to heat entry through its external heat exchanger, which is seen from the decrease in the warming phase time by almost half, 249 to 135 minutes, when the thermal diode is left in place. Thus, a thermal diode is necessary to decouple the heat sink/exchanger from the Al block when the TEC is off, thereby preventing residual heat transfer from the hot side of the TEC to the vaccines.

To increase the first warming time cool packs were employed as a phase change material; this increases the thermal inertia of the cooler (Sharma, Tyagi, Chen, \& Buddhi, 2009) because additional heat must be supplied from the environment to supply the cool pack's latent heat of fusion. However, the first and subsequent cooling times will also be increased because the additional heat must be removed by the TEC to freeze the cool packs.

The experimental success of this prototype vaccine cooler at high temperatures suggests that the cooler would be able to effectively transport vaccines in harsh environments. For example, with an estimated working time of over 10 hours, the cooler could keep vaccines in the range of $4-8^{\circ} \mathrm{C}$ for the duration of a workday with an ambient temperature of up to $37^{\circ} \mathrm{C}\left(98.6^{\circ} \mathrm{F}\right)$, temperatures typical of sub-Saharan Africa (UN FAO, 2012). Since maximum storage times will only increase as the ambient temperature decreases from $37^{\circ} \mathrm{C}$, this cooler could, in principle, be used in most areas across the world. Moreover, improvements in insulation, battery size/efficiency, and removing heat leaks coming from the ambient environment will increase the vaccine storage time.

Feasibility of onboard battery charging by solar panels

Initial testing showed that the panels would be able to recharge the two smaller 2000mAh lithium-ion batteries. However, the solar 


\begin{tabular}{lc}
\hline Average Warming Phase 1 $(\min \pm \mathrm{SD})$ & $249.3 \pm 21.8(n=7)$ \\
\hline Average Warming Phase 2 $(\min \pm \mathrm{SD})$ & $64.9 \pm 2.4(n=8)$ \\
\hline $\begin{array}{c}\text { Average Cooling Phase }(\min \pm \mathrm{SD}) \\
\text { Average Estimated Battery Charge Con- } \\
\text { sumption for Cooling Phase (mAh) }\end{array}$ & $\sim 4.700$ \\
\hline $\begin{array}{c}\text { Average Battery Charge Consumption } \\
\text { for Cooling Phase (\% of total capacity) }\end{array}$ & 20.4 \\
\hline
\end{tabular}

Table 1. Average warming and cooling times.*

\begin{tabular}{ccccc}
\hline & \multicolumn{2}{c}{ Trial 1 } & \multicolumn{2}{c}{ Trial 2 } \\
$\begin{array}{c}\text { Time } \\
(\mathbf{m i n})\end{array}$ & Voltage (V) & Current (A) & Voltage (V) & Current (A) \\
\hline 0 & 14.31 & 0.95 & 13.84 & 0.70 \\
\hline 10 & 14.21 & 0.82 & 14 & 0.74 \\
\hline 20 & 14.21 & 0.92 & 13.87 & 0.60 \\
\hline 30 & 14.18 & 0.91 & 13.82 & 0.47 \\
\hline 40 & 14.2 & 0.70 & 13.82 & 0.40 \\
\hline 50 & 14.37 & 0.83 & 13.97 & 0.53 \\
\hline 60 & 14.23 & 0.54 & 14.06 & 0.60 \\
\hline 70 & & & 14.01 & 0.47 \\
\hline 80 & & & 13.95 & 0.45 \\
\hline 90 & & & 13.96 & 0.47 \\
\hline 100 & & & 13.98 & 0.42 \\
\hline 110 & & & 14.02 & 0.35 \\
\hline
\end{tabular}

Table 2. Current and voltage delivered to the $2000 \mathrm{mAh}$ batteries using two solar panels as a function of time.**

*Although more than 25 experiments were performed to optimize conditions, the final first warming phase times reported in Table 1 are compromised of the average of 7 experiments and the final second warming and cooling phase times are comprised of an average of 8 experiments.

** Two 0.5 A-max solar panels connected in parallel.

panels would not be able to charge the larger battery powering the TEC. The solar panels are extremely sensitive to the orientation with respect to the sun (data not shown). Even in the most optimal solar conditions, the panels could produce only $1 \mathrm{~A} /$ hour. In the $\sim 5$ hours before the second warming phase, $\sim 6000 \mathrm{mAh}$ could be supplied to the battery making up the capacity lost in delivering charge to the TEC. However, because the second warming phase is considerably shorter, only about one-quarter of consumed charge could be replaced by the solar panels, not enough to independently sustain a second cooling phase. Thus, solar panel battery charging, while attractive in concept, does not seem practical because of the added cost and weight of the panels. This is especially true considering the capacity of Li-ion battery packs, which appear to be more than adequate as a power source for one eight-hour delivery trip and are fully rechargeable at the regional health center prior to the next trip to the field. The voltage and current data delivered to the $2000 \mathrm{mAh}$ batteries using two solar panels over time is shown in Table 2.

\section{Future Directions}

Cooler design

Future TE vaccine cooler designs will need to conform to require-ments of the forthcoming World Health Organization (WHO) prequalification specifications for active short-range vaccine cool-ers. The key among these is the need for the vaccine cooler to carry multiple vaccines with different packaging and the fact that initial cooling must be done onboard, presumably using regional health center power, because refrigerators in regional health cen-ters will not be able to accommodate the entire cooler. Note that in the current study, the vaccine cooler was brought to the starting temperature of $\sim 4^{\circ} \mathrm{C}$ in a laboratory refrigerator and Peltier cool-ing was employed only to hold the temperature in the range $4-8^{\circ} \mathrm{C}$ in an ambient of $37^{\circ} \mathrm{C}$ by turning on the thermoelectric chip when the temperature of the vaccines reached $8^{\circ} \mathrm{C}$.

\section{Thermal diode and heat exchanger}

In the present design, heat from the hot side of the TEC must be transferred to a remote heat exchanger. This requires an electri-cally powered pump and fan. To prevent backflow of the removed heat into the vaccine compartment, a thermal diode is required. The current cooler design employs a thermal diode that consists of a manually operated copper slab that must be inserted or with-drawn from the heat path; when in place the slab must make good thermal contact with both the hot side of the TE device and heat sink. There are other passive thermal diodes not requiring a man-ual operation such as the jumping drop thermal diode (Boreyko, Zhao, \& Chen, 2011; Weidenheft et al., 2017); however, at this juncture, they are difficult to mass produce. For field operation, the manual thermal diode combined with an additional electrical-ly powered pump and fan to transport heat to the environment is cumbersome at best.

The most desirable option would be to have a passive device to simultaneously remove heat while preventing backflow of removed heat into the vaccine compartment. Such heat pumping devices do exist in the form of vapor chambers or thermosiphons, which have the property of offering a simplified heat transfer solu-tion with no additional active components while simultaneously acting as thermal diodes (Bucci, 2017).

In summary, thermoelectrically cooled short-range vaccine coolers appear to be poised to make a major contribution to trans-porting viable vaccines to clients at the end stage of the cold with hermoelectric systems. Science, 321(5895), 1457-1461. doi:10.1126/ cold chanin: 1158899

Boreyko J. B. Zhao, Y., \& Chen, C. (2011). Planar jumping-drop thermal diodes. REFAP\&Ed Yhistcs Letters, 99(23), 234105. doi:10.1063/1.3666818

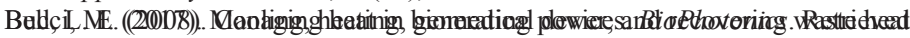

from: https://www.photonics.com/a61550/Managing_Heat_in_Biomedi cal Devices

Caspard, H., Coelingh, K. L., Mallory, R. M., Ambrose, C. S. (2016). Association 
of vaccine handling conditions with effectiveness of live attenuated influenza vaccine against $\mathrm{H} 1 \mathrm{~N} 1 \mathrm{pdm} 09$ viruses in the United States. Vaccine, 34(42), 5066-5072. doi:10.1016/j.vaccine.2016.08.079

Chatterjee, S., \& Pandey, K. (2003). Thermoelectric cold-chain chests for storing/transporting vaccines in remote regions. Applied Energy, 76(4), 415-433. doi:10.1016/s0306-2619(03)00007-2

Chen, D., \& Kristensen, D. (2009). Opportunities and challenges of developing thermostable vaccines. Expert Review of Vaccines, 8(5), 547-557. doi:10.1586/erv.09.20

Dieleman, J., Murray, C., \& Haakenstad, A. (2015). Financing global health 2014: Shifts in funding as the MDG era closes. Seattle, WA: Institute for Health Metrics and Evaluation.

Food and Agriculture Organization of the United Nations. (2012). The State of Food and Agriculture. FAO. ISSN 0081-4539

Gates, B. (2012). Annual Letter from Bill Gates. The Bill and Melinda Gates Foundation.

Ghoshal, U., \& Guha, A. (2009). Efficient switched thermoelectric refrigerators for cold storage applications. Journal of Electronic Materials, 38(7), 11481153

Humphreys, G. (2011). Vaccinations: rattling the supply chain. Bulletin of the World Health Organization, 89, 324-325

Larson, H. J., Cooper, L. Z., Eskola, J., Katz, S. L., \& Ratzan, S. (2011). Addressing the vaccine confidence gap. The Lancet, 378(9790), 526-535

Leng, F., Pecht, M., Tan, C.M. (2015). Effect of temperature on the aging rate of Li-ion battery operating above room temperature. Scientific Reports, 5(12). doi: $10.1038 /$ srep 12967

Matthias, D. M., Robertson, J., Garrison, M. M., Newland, S., Nelson, C. (2007). Freezing temperatures in the vaccine cold chain: A systematic literature review. Vaccine, 25(20), 3980-3986. doi:10.1016/j.vaccine.2007.02.052

Ohara, B., Sitar, R., Soares, J., Novisoff, P., Nunez-Perez, A., \& Lee, H. (2014). Optimization strategies for a portable thermoelectric vaccine refrigeration system in developing communities. Journal of Electronic Materials, 44(6), 1614-1626. doi:10.1007/s11664-014-3491-9

Putra, N. (2009). Design, manufacturing and testing of a portable vaccine carrier box employing thermoelectric module and heat pipe. Journal of Medical Engineering and Technology, 33(3), 232-237

Schoub, B. D., \& Cameron, N. A. (1996). Problems encountered in the delivery and storage of OPV in an African country. Dev. Biol. Stand., 87, 27-32

Sharma, A., Tyagi, V.V., Shen, C.R., Buddhi, D. (2009). Review on thermal energy storage with phase change materials and applications. Renewable and Sustainable Energy Reviews, 13(2), 318-345. doi: 10.1016/j.rser.2007.10.005

UNICEF. (2011). UNICEF Supply Annual Report. Copenhagen. UNICEF Supply Division.

Wallace, A. S., Willis, F., Nwaze, E., \& Dieng, B. (2017) Vaccine: Vaccine wastage in Nigeria: An assessment of wastage rates and related vaccinator knowledge, attitudes and practices. Vaccine, 35(48), 6751-6758 doi:10.1016/j.vaccine.

Wiedenheft, K. F., Guo, H. A., Qu, X., Boreyko, J. B., Liu, F., Zhang, K., ... Chen, C. (2017). Hotspot cooling with jumping-drop vapor chambers. Applied Physics Letters, 110(14), 141601. doi:10.1063/1.4979477

Zaffran, M., Vandelaer, J., Kristensen, D., Melgaard, B., Yadav, P., Antwi-Agyei, K. O., \& Lasher, H. (2013). The imperative for stronger vaccine supply and logistics systems. Vaccine, 31, B73-B80 